\title{
Structural and Electronic Instabilities in Polyacenes: Density Matrix Renormalization Group Study of a Long-Range Interacting Model
}

\author{
C. Raghu ${ }^{1}$, Y. Anusooya Pati ${ }^{2}$ and S. Ramasesha ${ }^{3}$ \\ Solid State and Structural Chemistry Unit \\ Indian Institute of Science, Bangalore 560 012, India
}

\begin{abstract}
We have carried out Density Matrix Renormalization Group (DMRG) calculations on the ground state of long polyacene oligomers within a Pariser-Parr-Pople (PPP) Hamiltonian. The PPP model includes long-range electron correlations which are required for physically realistic modeling of conjugated polymers. We have obtained the ground state energy as a function of the dimerization $\delta$ and various correlation functions and structure factors for $\delta=0$. From energetics, we find that while the nature of the Peierls' instability in polyacene is conditional and strong electron correlations enhance the dimerization. The cis form of the distortion is favoured over the trans form. However, from the analysis of correlation functions and associated structure factors, we find that polyacene is not susceptible to the formation of a bond order wave (BOW), spin density wave (SDW) or a charge density wave (CDW) in the ground state.
\end{abstract}

PACS numbers : 31.25.Qm, 71.10.Fd, 71.30.+h, 71.45.-d 


\section{Introduction}

The question whether an infinitely long linear polyene (polyacetylene, PA) would have equal bond lengths or not has been debated ever since it was known that benzene has equal bond lengths while 1,3,5-hexatriene has alternating short (double) and long (single) bonds. Addressing this issue, Lennard-Jones [1] and Coulson [2] predicted a ground state with uniform bond lengths. Later work by Labhart [3], Ooshika [4], Longuet-Higgins and Salem [5] established that the ground state would have bond-alternation i.e. the infinite polyene would have alternate long and short bonds. Experimentally, such a dimerization would qualitatively explain the finite optical gap obtained by extrapolation of optical data for linear polyenes [6]. Earlier, Peierls established a more general result, known as the Peierls' instability or Peierls' distortion [7] for a partially filled one-dimensional band. Peierls' demonstrated that lattice vibrations couple to electrons in the band leading to the opening of a gap at the Fermi surface, making the material insulating. The Hückel model solution of Longuet-Higgins and Salem pertain to the specific case of Peierls' distortions in a half-filled extended system. Longuet-Higgins and Salem showed that for an infinite polyene chain, the total electronic energy per carbon atom as a function of the distortion $\delta$ in the chain, within a Hückel model [8] is proportional to $\delta^{2} \ln |\delta|[5]$. The elastic strain energy of the system is proportional to $\delta^{2}$, the proportionality constant depending directly on the lattice stiffness and inversely on the electron-phonon coupling constant. The algebraic forms of the strain and electronic energies guarantee that the gain in electronic energy always exceeds the strain energy and the ground state would correspond to nonzero $\delta$. The distortion of the polyene chain is termed unconditional as the distorted state is always more stable than the undistorted state for any value of the 
lattice stiffness and the electron-lattice coupling constant. In the last two to three decades, a lot of interest has been generated by the possibility of solitonic and polaronic excitations in polyacetylene (PA) following the work of Pople and Walmsley [9], and later by Rice [10] and Su, Schrieffer and Heeger [11]. The latter work resulted in the Su-SchriefferHeeger (SSH) model for polyacetylene.

All the work described above was done in the context of non-interacting quantum cell models. However, real materials have interacting electrons and the importance of including electron-electron interactions has been demonstrated while interpreting the ordering of excited states in polyenes [12]. The correct ordering, namely the $2^{1} A_{g}$ state lying below the optically allowed $1^{1} B_{u}$ state in long polyenes can be obtained only in a correlated electronic model. The effect on dimerization in the ground state of polyacetylene in a correlated model was unclear till the real-space, valence-bond (VB) study by Mazumdar and Dixit [13] for Hubbard model and Soos and Ramasesha for PPP model [14]. While mean-field and Hartree-Fock approaches predicted a decrease in dimerization, Mazumdar and Dixit found a clear enhancement, over a fairly large range of correlation strength with a maximum dimerization around $U \sim 4 t$. In their study they employed a model that included electron correlation at a minimal level, namely the Peierls'-Hubbard model. This result was subsequently verified by quantum Monte Carlo study by Hirsch [15], variational calculation by Baeriswyl and Maki [16] and numerical renormalization group study by Hayden and Mele [[17]. Soos and Ramasesha [14] found a similar result in case of PPP model which includes extended range electron correlation. Thus it is now widely believed that electron correlations enhance dimerization in the Peierls-Hubbard model.

The Peierls' instability is mainly a one-dimensional phenomena since only in one- 
dimension does the Peierls' gap open along the full Fermi surface. In higher dimensions, Peierls' instability is not realized as easily as in one-dimension [7]. The effect of dimensionality on the extent of instability has been studied within the frame work of energy band theory, where it is held that the strength of the instability depends on the extent of nesting of the Fermi surface. The effect of dimensionality on the instability, in going from one-dimension to quasi one-dimension can be explored by studying coupled chain (ladders) systems. The realm of quasi one-dimension is interesting as it provides for interplay of strong quantum fluctuations as seen in one-dimension on the one hand and possibility of charge or spin to order as seen in two-dimensions on the other. Historically, ladders have been investigated within the Hubbard model mainly to explore instabilities like pairing instability, charge and spin gaps [18], or charge ordering phenomenon [19]. The experimental realization of these systems are the two-legged $\mathrm{SrCu}_{2} \mathrm{O}_{3}$ and the threelegged $\mathrm{Sr}_{2} \mathrm{Cu}_{3} \mathrm{O}_{5}$ ladder compounds [20] wherein the spin exchange or electron transfers are confined to a pair of parallel chains.

In the realm of conjugated polymers, polyacenes (Fig. 1a) can be viewed as ladders with missing alternate rungs. These systems can be fairly easily realized experimentally. Polyacene molecules with up to seven rings have already been synthesized in the laboratory. Recently, polyacenes received a lot of attention when tetracene was used as the lasing material to make the first electrically pumped organic laser [21]. It was also demonstrated that one could make very high quality field-effect transistors (FETs) based on pentacene. These devices display exotic behaviour like fractional quantum hall effect, superconductivity and electrical switching in the FET configuration [22]. Quantum chemists have been interested in polyacenes for a very long time. Early studies on Peierls' instability in polyacenes were carried out by Salem and Longuet-Higgins within the Hückel model 
[23]. They observed that unlike in the case of PA, instability in polyacene is conditional; it depends on the lattice stiffness for a given electron-phonon coupling. In PA, the distorted state is more stable than the undistorted state for any value of the force constant and electron-phonon coupling. Salem and Longuet-Higgins considered the cis distorted form (see Figs. 1b and 1c) while Boon [24] argued that the trans form was the more stable form of distortion. Subsequent studies by Whangbo, Woodward and Hoffmann [25], and Tanaka et al. [26] added to this claim. Studies including electron correlations were initially confined to the mean-field picture. Kivelson and Chapman [27] studied bond alternation, magnetic ordering and possible superconductivity in polyacenes. In addition, there have been various other studies on the origin of the band gap, metal-insulator transition and spin-Peierls' distortions [28]. The antiferromagnetic spin-1/2 Heisenberg system with nearest neighbour exchange corresponding to polyacene geometry has been studied by Garcia-Bach, Valenti and Klein to explore the possibility of spin-Peierls' instability in the system [29].

Studies of Peierls' instability using quantum cell models with explicit electron correlations in the context of polyacenes have been very few and these investigations have employed only the Hubbard model. A modified Gutzwiller variational study by O'conner and Watts-Tobins [30] reaffirmed the conditional nature of the instability. The instability investigated by employing the Projector Quantum Monte Carlo technique (PQMC) for finite oligomers of polyacenes concluded the same [31]. This study also found that electron correlations enhance the susceptibility to distortion. Besides, for large interaction strength, the undistorted polyacene was found to show a tendency for formation of a SDW ground state. 
Polyacenes, being semiconducting, the electron-electron interactions are long ranged. The QMC technique is difficult to implement for quantum cell models with long range interactions. The Hubbard-Stratanovich transformation of each interaction term leads to a bosonic variable in QMC. Thus, even for small quantum cell systems, the number of bosonic variables become prohibitively large. Besides, the QMC method is also restricted to intermediate correlation strengths. In our present study of instability in polyacene, we have used a Pariser-Parr-Pople (PPP) model and have employed the Density Matrix Renormalization Group (DMRG) method [32] to solve the model. The PPP model includes long-range coulomb interactions and appears unsuitable for a DMRG study as it apparently spoils the quasi-one-dimensional topology of the system. However, our earlier studies on PA has shown that the DMRG method is quite accurate for the PPP model [35] and it appears that the DMRG method retains its accuracy if the long-range interaction part is diagonal, as is indeed the case in the PPP model. In this paper we first review the results obtained from a non-interacting model, then present our results and discussion on polyacene and finally a summary of the results.

\section{The non-interacting picture}

The analysis by Salem and Longuet-Higgins [23] was based on a Hückel model study of polyacene. They considered an infinitely long chain of polyacene as made of two infinite polyenes joined together by cross links (rungs), as shown in Fig. 1a. The molecular orbitals (MOs) of each unit cell of polyacene may be classified as symmetric or antisymmetric according to their symmetry with respect to reflection about the plane bisecting the rung bonds, indicated in the figure by a dashed line. There are in all four bands, 
arising from the four $\pi-\mathrm{MOs}$ in each unit cell, two of each symmetry type as shown in Fig. 2. In the undistorted form of polyacene, there is no band gap between the occupied and empty bands, while in both the cis and trans distorted forms (Figs. 1b and 1c) there is gap between the occupied and empty bands. In the undistorted case, since the lower two bands are completely filled and the upper two bands are empty, we expect the system to be insulating. However, the absence of a band gap between the filled lower band and the empty higher band, due to accidental degeneracy, makes uniform polyacene a rather unconventional metal. If a symmetrical distortion potential is imposed by way of a dimerization one might expect this degeneracy to be lifted. However, the matrix element of the perturbation between the symmetrical and antisymmetrical states at the band edge vanish by symmetry. Thus the case of polyacene, even in the noninteracting limit, is different from PA and we cannot conclude if we will observe a dimerization of the chain. Salem and Longuet-Higgins [23] conjectured that the polyacene would show only a conditional instability.

Kivelson and Chapman [27], based on their study of polyacene by a nearest-neighbour tight binding model, argue that since the energy of the states near the Fermi energy is a quadratic function of the wave vector $k$ (rather than linear as in PA) the density of states diverge near the Fermi energy, enhancing the possibility of instabilities in polyacene. Their study arrives at the conclusion that there is no structural instability of the two kinds depicted in Figs. 1a and 1b. They speculate on the possibility of a superconducting or a magnetically ordered ground state. They however ignore electron-electron interactions by considering them as "probably weak". 


\section{The PPP model and Computational Scheme}

The PPP model has been widely studied in the context of conjugated polymers and molecules [36]. The PPP model Hamiltonian is given by,

$$
\begin{gathered}
\hat{H}_{\mathrm{PPP}}=\hat{H}_{0}+\hat{H}_{\mathrm{int}} \\
\hat{H}_{0}=\sum_{l=1}^{2} \sum_{i, \sigma} t_{0}\left[1+\eta_{l}(-1)^{i} \delta\right]\left(\hat{a}_{i, l \sigma}^{\dagger} \hat{a}_{i+1, l \sigma}+H . c .\right)+\sum_{i, \sigma} t_{0}\left(\hat{a}_{2 i-1,1 \sigma}^{\dagger} \hat{a}_{2 i-1,2 \sigma}+H . c .\right), \\
\hat{H}_{\text {int. }}=\sum_{i} \sum_{l} \frac{U_{i l}}{2} \hat{n}_{i, l}\left(\hat{n}_{i, l}-1\right)+\sum_{i, j} \sum_{l, m} V_{i l, j m}\left(\hat{n}_{i, l}-1\right)\left(\hat{n}_{j, m}-1\right) .
\end{gathered}
$$

where $l, m$ are the chain index, and $i, j$ refer to sites on a chain, $\hat{a}_{i, l \sigma}^{\dagger}\left(\hat{a}_{i, l \sigma}\right)$ creates (annihilates) an electron of spin $\sigma$ at site $i$ on chain $l, t_{0}$ is the transfer integral, which alternates between $t_{0}(1+\delta)$ and $t_{0}(1-\delta)$ for adjacent bonds on the same chain, $\delta$ being the dimensionless dimerization parameter, $\eta_{1}=\eta_{2}=1$ if the dimerization of the top $(l=1)$ chain is in phase with the dimerization of the bottom chain $(l=2)$ (cis configuration) and $\eta_{1}=1, \eta_{2}=-1$ if the dimerization of the top chain is out of phase with the dimerization of the bottom chain (trans configuration). $U_{i l}$ is the on-site Hubbard interaction and $V_{i l, j m}$ is the intersite Coulomb interaction, interpolated between $U_{i, l}$ and $e^{2} / r, r \longrightarrow \infty$, using the Ohno interpolation scheme [37],

$$
V_{i l, j m}=14.397\left[\frac{28.794}{\left(U_{i l}+U_{j m}\right)^{2}}+r_{i l, j m}^{2}\right]^{-\frac{1}{2}}
$$

(the distances are in $\AA$ and the energies in $\mathrm{eV}$ ) widely used for conjugated polymers. In all our computations, we have used the standard PPP parameters for carbon, which is $U=11.26 \mathrm{eV}, t_{0}=2.4 \mathrm{eV}$ for bond length $r=1.397 \AA$ which corresponds to $\delta=0$. In 
computing distances we have assumed that all bond angles are $120^{\circ}$ and that bond lengths scale as $r(1-\delta)$ for a bond with transfer integral $t=t_{0}(1+\delta)$.

The DMRG method, invented by White [32] is the most accurate numerical method yet for calculating the ground and low-lying states of interacting quasi-one-dimensional systems. In our application of this technique to polyacenes within the PPP model, there are two crucial differences from all the earlier implementations of the technique for lowdimensional systems. These are (i) the interaction part, although diagonal in the real space representation, is truly long-ranged and (ii) the topology of the system being constructed does not have structures corresponding to oligomers of polyacene at every stage of the DMRG iteration. Incorporating long-ranged interaction implies that we need to renormalize and store the matrices corresponding to the number operators of all the sites at each iteration. While this is quite straightforward, it takes up large storage and for efficiency of computations we have stored them in sparse form. Typically only about $5 \%$ of the matrix elements of the site number operators are nonzero.

The nonlinear topology of polyacenes implies that we should find an efficient and accurate way of building the oligomers. We should avoid using cyclic boundary conditions or long range transfer operators in building the final system. In Fig. 3 we show schematically the way the polyacene oligomers are constructed in the DMRG scheme. We begin with a ring of four sites and systematically add new sites in the middle of the system building up the molecule in such a way that we avoid (i) long-range transfers between new and old sites and (ii) transfers between old sites that are far apart. We have extensively checked this procedure by carrying out calculations on large oligomers in the noninteracting limit and comparing them with exact numerical results. 
We have performed both infinite and finite DMRG calculations on polyacene chains with up to 24 monomer units. We have done DMRG calculation with 128 density matrix eigenvectors after making sure that the energies converge for this truncation, by calculating for a few oligomers with larger cut-offs. Besides, the ground state energy for napthalene and anthracene obtained from DMRG with a cut-off of 128 compare very well with model exact calculations in a Valence Bond (VB) basis [33, 34]. Symmetries like conservation of $z$-component of total spin $S_{z}$ and total number of electron $N_{\text {tot }}$ have been exploited in our implementation. Since the left block-right block reflection symmetry commonly seen in DMRG calculations is broken due to the bond alternation in each chain, we have to store relevant operators for every site on both the left and right blocks. This doubles the storage but can be easily handled as all the site operators are highly sparse. Properties like expectation value of observables or correlation functions can be evaluated with great accuracy after a few iterations of finite system DMRG algorithm.

\section{Results and discussion}

Our studies are divided into two parts: (1) study of the dependence of the energy of the system on $\delta$, and (2) study of susceptibility of the ground state to instabilities for $\delta=0$ as seen from appropriate structure factors. We approach the question of Peierls' distortion first on the basis of energetics. We have calculated the total energy of the system as a function of the system size $N$ and dimerization $\delta$, up to a maximum system size of 24 monomer units for $\delta$ values ranging from 0.01 to 0.1 by employing the infinite system DMRG algorithm. In Fig. 4 we show the dependence of the energy per unit cell on the inverse system size, $1 / N$. We note that this dependence is linear in $1 / N$. In the case of the 
trans polyacene, oligomers with even and odd number of unit cells fall on two different straight lines for large $\delta$. The smooth behaviour of the energy per unit cell versus $1 / N$ gives confidence in extrapolation of the enegries to the thermodynamic limit.

In order to study the nature of the structural instability, if any, in the polyacenes, we study the stabilization energy for the distorted structure, $\Delta E_{A}(N, \delta)=E(N, 0)-E_{A}(N, \delta)$ where $A=C$ or $T$ corresponding to cis or trans form of polyacene, for various $\delta$ and number of rings, $N$ in the polyacene. By definition, positive values of $\Delta E_{A}$ would indicate that the distorted structure is stabilized. We obtain the stabilization energy in the thermodynamic limit, $\Delta E_{A}(\infty, \delta)$, for each value of $\delta$ from $\delta=0.01$ to 0.1 by extrapolation of $\Delta E_{A}(N, \delta) / N$ versus $1 / N$. In Fig. 5 we present the variation of $\Delta E_{A}(\infty, \delta)$ with $\delta$. Also shown for comparison are the Hückel results for this system. We note that the stabilization of the dimerized state in the interacting system is larger than the stabilization of the same in the noninteracting model for both cis and trans distortions. This shows that dimerization is favoured in the interacting models more than in the noninteracting models, just as in the case of polyenes. From the nature of the curve in Fig. 5, the stabilization energy appears to be quadratic in $\delta$, for both cis and trans form of polyacene. The plot of $\Delta E_{A}(\infty, \delta) v s \delta^{2}$ shown in the inset, in fact shows that the stabilization is quadratic in $\delta$ to a very good approximation. The quadratic form of the electronic stabilization of the dimerization implies that the instability in polyacenes is indeed conditional for both the cis and the trans forms. This is in contrast to the unconditional dimerization seen in PA. Furthermore, it is also seen that for the same distortion $\delta$, the cis form is more susceptible than the trans form to dimerization. Earlier work [31] within a Hubbard model also found that the cis form is stabilized more than the trans form in the presence of electron-electron interactions. The long range nature of interactions considered here does not seem to affect 
this result. Indeed, even the Hückel model also shows that the cis form is stabilized more than the trans form.

To get a physical picture of the ground state, we have studied many different types of correlation functions. In the context of a structural instability like the formation of a bondorder wave (BOW), the bond-bond correlation function of the undistorted system gives information about the type of BOW instability that may occur in the system. The Fourier transform of the bond-bond correlation function, the bond structure factor, gives the amplitude for various BOW instabilities that may be present in the ground state. Similarly, the structure factors associated with charge-charge and spin-spin correlation functions provide information on the susceptibility of the system towards CDW and SDW instabilities. We have calculated bond-bond, spin-spin and charge-charge correlation functions in the ground state for twenty four ring polyacene system which is the largest system size that we have been able to study. A unit cell of polyacene has five bonds as shown in Fig. 6a; of these the bonds 1, 2, 4 and 5 are important to characterize a distortion as either cis or trans. It is possible to construct five different types of bond-bond correlation functions with respect to a reference bond. From our view point, the important correlation functions involve bond 1 with bonds 1 and 2 in each unit cell as well as bond 1 with bonds 4 and 5 in each unit cell. The first type of correlation function is useful in understanding if the polyacenes indeed distort while the second type are useful in identifying the type of distortion if it is present.

The bond-bond correlation function is defined as

$$
<\hat{b}_{i, l} \hat{b}_{j, l^{\prime}}>=<\left(\sum_{\sigma} \hat{a}_{i, l \sigma}^{\dagger} \hat{a}_{i+1, l \sigma}+H . c .\right)\left(\sum_{\tau} \hat{a}_{j, l^{\prime} \tau}^{\dagger} \hat{a}_{j+1, l^{\prime} \tau}+H . c .\right)>
$$

where $l, l^{\prime}$ denote the chain. In the DMRG method, the expectation value of the product 
of two operators are very accurate, if they belong to different blocks. Thus, the bondbond correlations are calculated between the bond on one half with all the bonds on the other half. The bond operator is itself a product of two operators in the same block. We have computed the matrix of this product explicitly when it is first encountered and have renormalized the product matrix thereafter. The correlation functions are calculated for open polyacene chain. However, the resulting correlation function cannot be Fourier transformed since the system is not strictly periodic. To overcome this difficulty, we have assumed that the correlations involving the interior rings are identical with those computed for a periodic system (Fig. 6b). This is reasonable, if we neglect sites belonging to the two rings at each of the ends. Then the correlations corresponding to the properties of the interior twenty rings are taken to be the same as those in a twenty ring system with periodic boundary conditions. This allows obtaining structure factors from the corresponding correlation functions. To enhance the accuracy of our calculations, we have carried out finite DMRG calculations for the 24 ring polyacene system. We have kept the DMRG cut-off at 150 density matrix eigenvectors and have used four finite DMRG sweeps.

We show the plot of bond-bond correlations for the bond in the bottom chain (shown in bold in Fig. 6b) with all the chain bonds on the top and bottom left half of the system in Figs. $7 \mathrm{a}$ and $7 \mathrm{~b}$. We note that except at the ends of the chain, the correlations are almost identical. The bond-bond correlation shows a slight short range oscillation which dies off rapidly. Eliminating the end bonds and imposing periodicity as discussed earlier, we have obtained the structure factor corresponding to the bond-bond correlations. This is shown in Fig. 8. We note that the structure factor has no peaks anywhere away from $q=0$. This clearly implies that the system is not susceptible to any bond order wave. Since a single 
chain remains uniform, the question of cis or trans type of distortion does not arise. We have also confirmed that the bond-bond correlation for the rung bonds shows a similar behaviour, implying that the rung bonds are uniform.

The spin-spin correlation functions, $\left\langle s_{i, l}^{z} s_{j, l^{\prime}}^{z}>\right.$ and charge-charge correlation functions, $<n_{i, l} n_{j, l^{\prime}}>$ have also been computed to see if the system has a tendency for formation of either a SDW or CDW. According to Fig. 9a, there are four sites in a unit cell of undistorted polyacene. We have calculated these correlations between the new right site (Fig. 9b) and all the sites in the left block. The real space spin-spin correlations, displayed in Figs. 10a and 10b show short range antiferromagnetic fluctuations expected from a nondegenerate correlated model [38]. The spin-spin correlation decays exponentially which is consistent with a spin gap in the system. The charge-charge correlations, shown in Figs. 11a and 11b show very slight short range oscillation, extending over a couple of sites. They also decay rapidly. The structure factors for spin-spin and chargecharge correlations are shown in Figs. 12 and 13. They clearly show that the system favours uniform charge distribution and no spin density oscillations. This rules out any possibility of CDW or SDW ground state in polyacenes.

\section{Summary}

We have studied polyacenes up to 24 rings with long range coulomb interactions within a PPP model, by the DMRG method. From energetics, we conclude that the structural instability in polyacene is only conditional, unlike that in the case of PA. We find that the cis form of distorted polyacene is more stable than the trans form. This is contrary to earlier predictions based on non-interacting models where the trans form was predicted 
to be more favourable. We have used the finite DMRG algorithm to calculate correlation functions like bond-bond, spin-spin and charge-charge in the undistorted ground state. Analysis of these correlation functions and their associated structure factors leads to the conclusion that polyacene ground state does not have the tendency to show BOW, SDW or CDW instability.

Acknowledgements: We acknowledge financial support of CSIR (Project No. 1595/99/EMRII) and BRNS, Dept. of Atomic Energy, (Project No. 99/37/37/BRNS), India.

${ }^{1}$ raghu@sscu.iisc.ernet.in ; ${ }^{2}$ anusooya@sscu.iisc.ernet.in ; ${ }^{3}$ ramasesh@ sscu.iisc.ernet.in

\section{References}

[1] J. E. Lennard-Jones, Proc. Roy. Soc A 158, 280 (1937).

[2] C. A. Coulson, Proc. Roy. Soc A 164, 383 (1938).

[3] H. Labhart, J. Chem. Phys. 27, 957 (1957).

[4] Y. Ooshika, J. Phys. Soc. Jp. 12, 1238 (1957).

[5] Longuet-Higgins and Salem. L, Proc. Roy. Soc A 251, 172 (1959).

[6] Kuhn. H, Helv. Chim. Acta, 31, 1441 (1948).

[7] R. E. Peierls, Quantum Theory of Solids, Clarendon, Oxford (1955).

[8] E. Hückel, Z. Physik 70, 204 (1931); 76, 628 (1932).

[9] J. A. Pople and S. H. Walmsley, Mol. Phys. 5, 15 (1962).

[10] M. J. Rice, Phys. Lett. A 71, 152 (1979). 
[11] W. P. Su, J. R. Schrieffer and A. J. Heeger, Phys. Rev. Lett. 42, 1698 (1979); Phys. Rev. B 22, 2099 (1980).

[12] Z. G. Soos, S. Ramasesha and D. S. Galvao, Phys. Rev. Lett. 71, 1609 (1985).

[13] S. Mazumdar and S. N. Dixit, Phys. Rev. Lett. 51, 292 (1983); S. N. Dixit and S. Mazumdar, Phys. Rev. B 29, 1824 (1984).

[14] Z. G. Soos and S. Ramasesha, Phys. Rev. B 29, 5410 (1984).

[15] J. E. Hirsch, Phys. Rev. Lett. 51, 296 (1983).

[16] D. Baeriswyl and K. Maki, Phys. Rev. B 31, 6633 (1985).

[17] G. Hayden and E. Mele, Phys. Rev. B 32, 6527 (1985).

[18] S. Daul and D. J. Scalapino, Phys. Rev. B 62, 8658 (2000); E. Jeckelmann, D. J. Scalapino and S. R. White, Phys. Rev. B 58, 9492 (1998).

[19] Matthias Vojta, Arnd Hübsch and R. M. Noack, Phys. Rev. B 63, 045105 (2001).

[20] M. Azuma, Z. Hiroi, M. Takano, K. Ishida and Y. Kitaoka, Phys. Rev. Lett. 73, 3463 (1994).

[21] J. Schön, Ch. Kloc, A. Dodabalapur and B. Batlogg, Science, 289, 599 (2000).

[22] J. Schön, Ch. Kloc and B. Batlogg, Science, 288, 2338 (2000); ibid. Nature, 406, 702 (2000); J. Schön, S. Berg, Ch. Kloc and B. Batlogg, Science, 287, 1022 (2000).

[23] L. Salem and H. C. Longuet-Higgins, Proc. Roy. Soc A 255, 435 (1960).

[24] M. R. Boon, Theor. Chim. Acta (Berl.), 23, 109 (1971). 
[25] M. -H. Whangbo, R. Hoffman, R. B. Woodward, Proc. Roy. Soc A 366, 23 (1979).

[26] K. Tanaka, K. Ozeki, S. Nankai, T. Yamabe and H. Shirakawa, J. Phys. Chem. Solids 44, 1096 (1983).

[27] S. Kivelson and O. L. Chapman, Phys. Rev. B 28, 7236 (1983).

[28] M. Baldo, A. Grassi, R. Pucci and P. Tomasello, J. Chem. Phys. 77, 2438 (1982); M. Baldo, G. Piccito, R. Pucci and P. Tomasello, Phys. Lett A 95, 201 (1983); T. Yamabe, K. Tanaka, K. Ozheki and S. Yata Sol. State. Comm 44, 823 (1982); A. K. Bakshi and J. Ladik, Ind. J. Chem. A 33, 494 (1994).

[29] M. A. Garcia-Bach, R. Valenti and D. J. Klein, Phys. Rev. B 56, 1751 (1997).

[30] M. P. O’Connor and R. J. Watts-Tobins, J. Phys. C: Solid State Phys. 21, 825 (1988).

[31] Bhargavi Srinivasan and S. Ramasesha, Phys. Rev. B 57, 8927 (1998).

[32] S. R. White, Phys. Rev. Lett 69, 2863 (1992); Phys. Rev. B 48, 10345 (1993)

[33] S. Ramasesha, G. S. Galvao and Z. G. Soos, J. Phys. Chem 97, 2823 (1993).

[34] S. Ramasesha and Z. G. Soos, Chem. Phys. 91, 35 (1984).

[35] S. Ramasesha and Kunj Tandon in Density-Matrix Renormalization: A New Numerical Method in Physics, Lecture notes in physics, Eds. I. Peschel, X. Wang, M. Kaulke and K. Hallberg, (Springer-Berlin) (1999), p. 247-260.

[36] For a review, see Z.G.Soos, D. Mukhopadhyay and S. Ramasesha, in Nonlinear Optical Materials: Theory and Modelling, Eds., S.P. Karna and A. T. Yeates, ACS 
Monograph, Washington, DC, (1996), p. 189-210; D. Baeriswyl, D. K. Campbell and S. Mazumdar, in Conjugated Conducting Polymers, Ed. H. G. Kiess, Springer-Verlag (1992), p. 7-134.

[37] K. Ohno, Theor. Chem. Acta 2, 219 (1964); G. Klopman, J. Am. Chem. Soc. 86, 4550 (1964).

[38] S. Ramasesha and Z.G. Soos, Phys. Rev., B 32, 5368 (1985). 


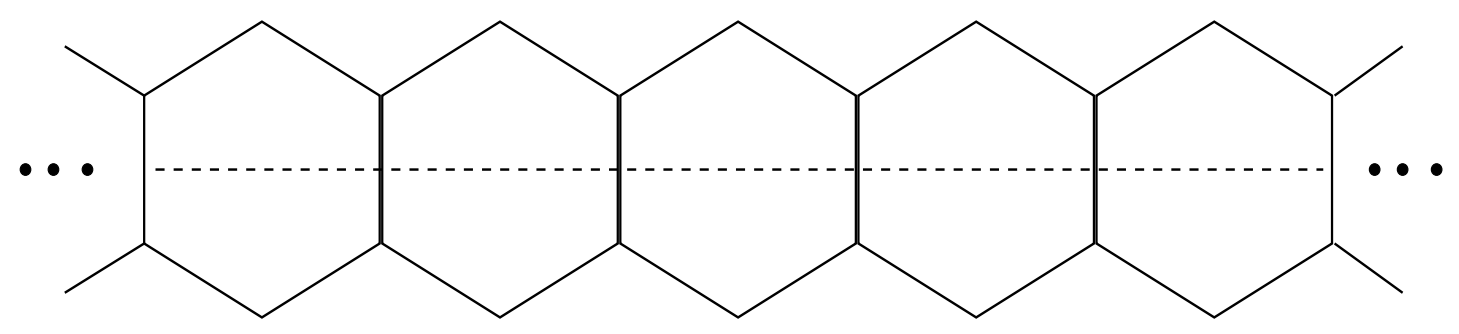

( a )

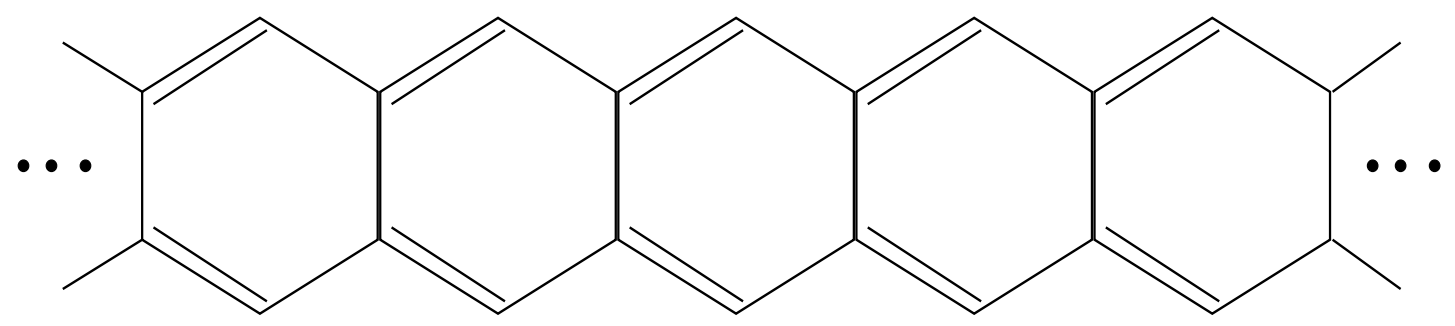

( b )

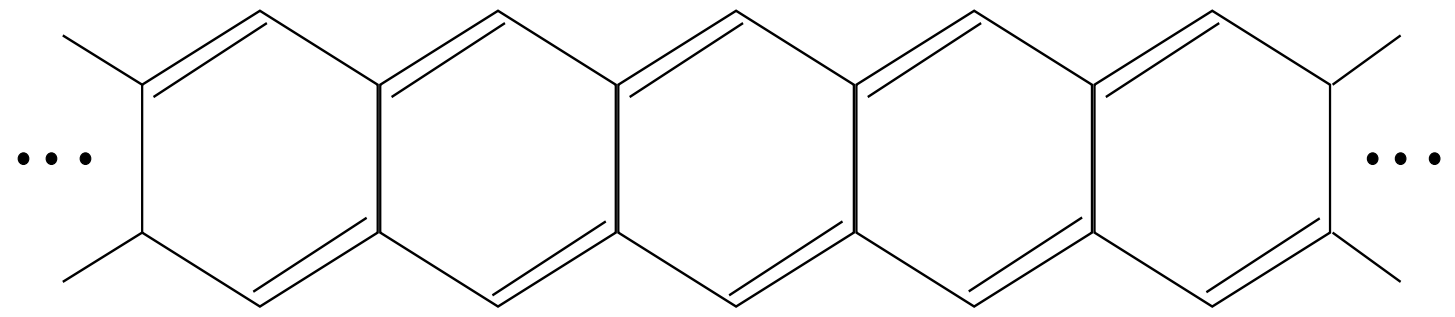

(c)

Figure 1: Structure of polyacene. (a) Undistorted or uniform, (b) Cis distorted form, (c) Trans distorted form. 


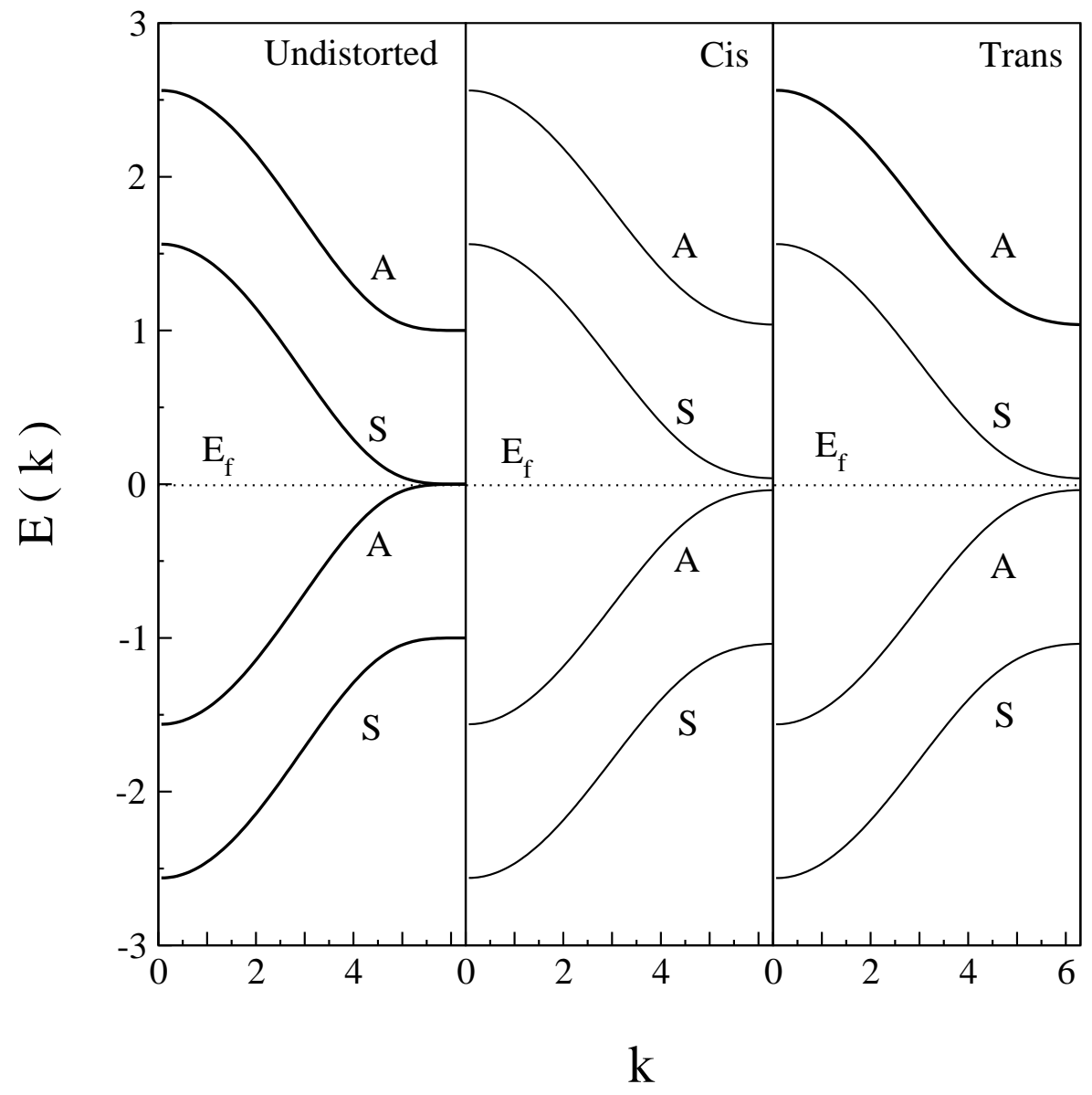

Figure 2: Dispersion of the $\pi$-bands in polyacene for the three forms. The cis and trans forms correspond to dimerization of $\delta=0.2$. 

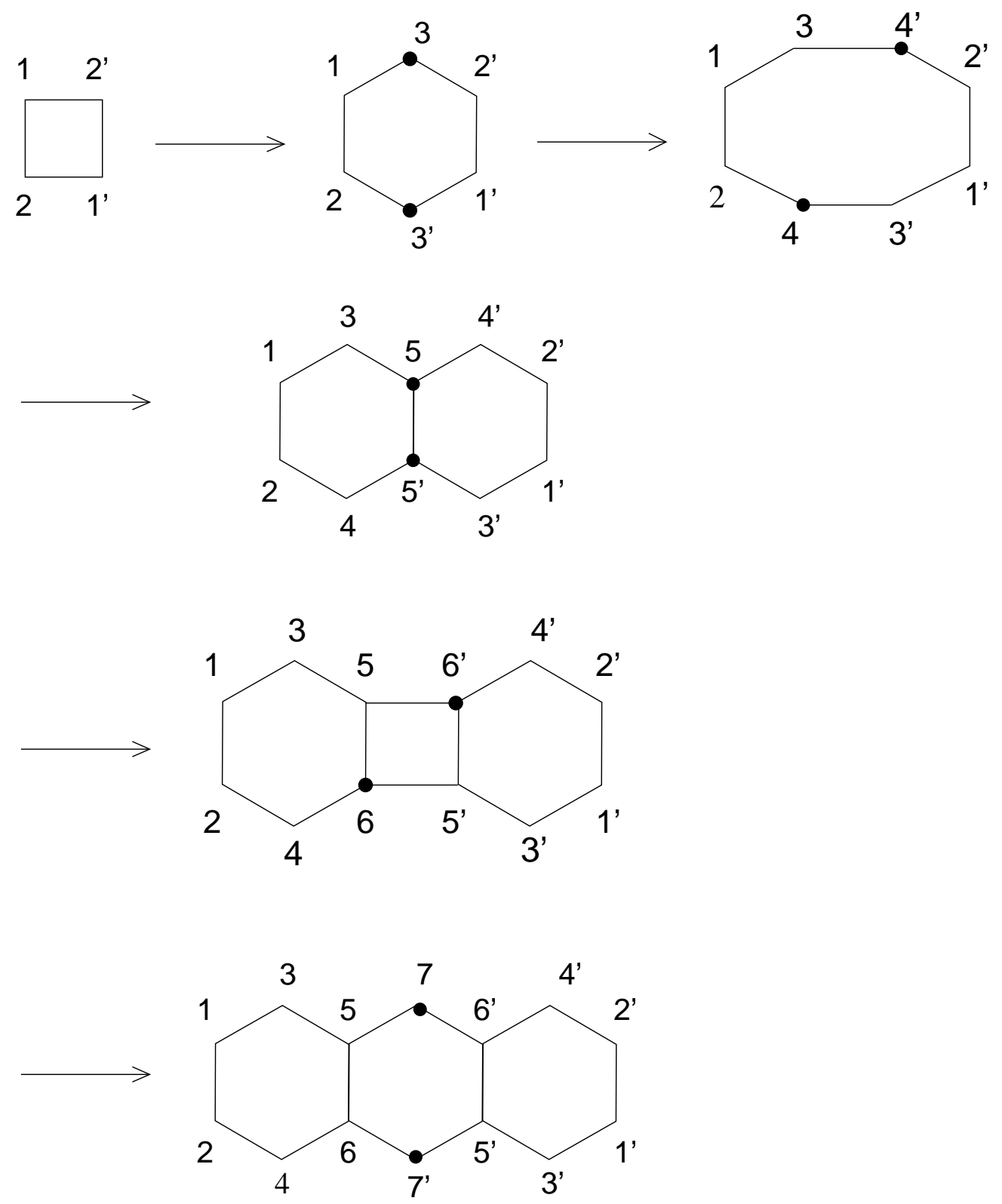

Figure 3: Inside-out scheme for building polyacene oligomers, adding two sites at a time in the DMRG procedure, starting from a four site system. The primed sites correspond to the right block and the unprimed sites correspond to the left block. 


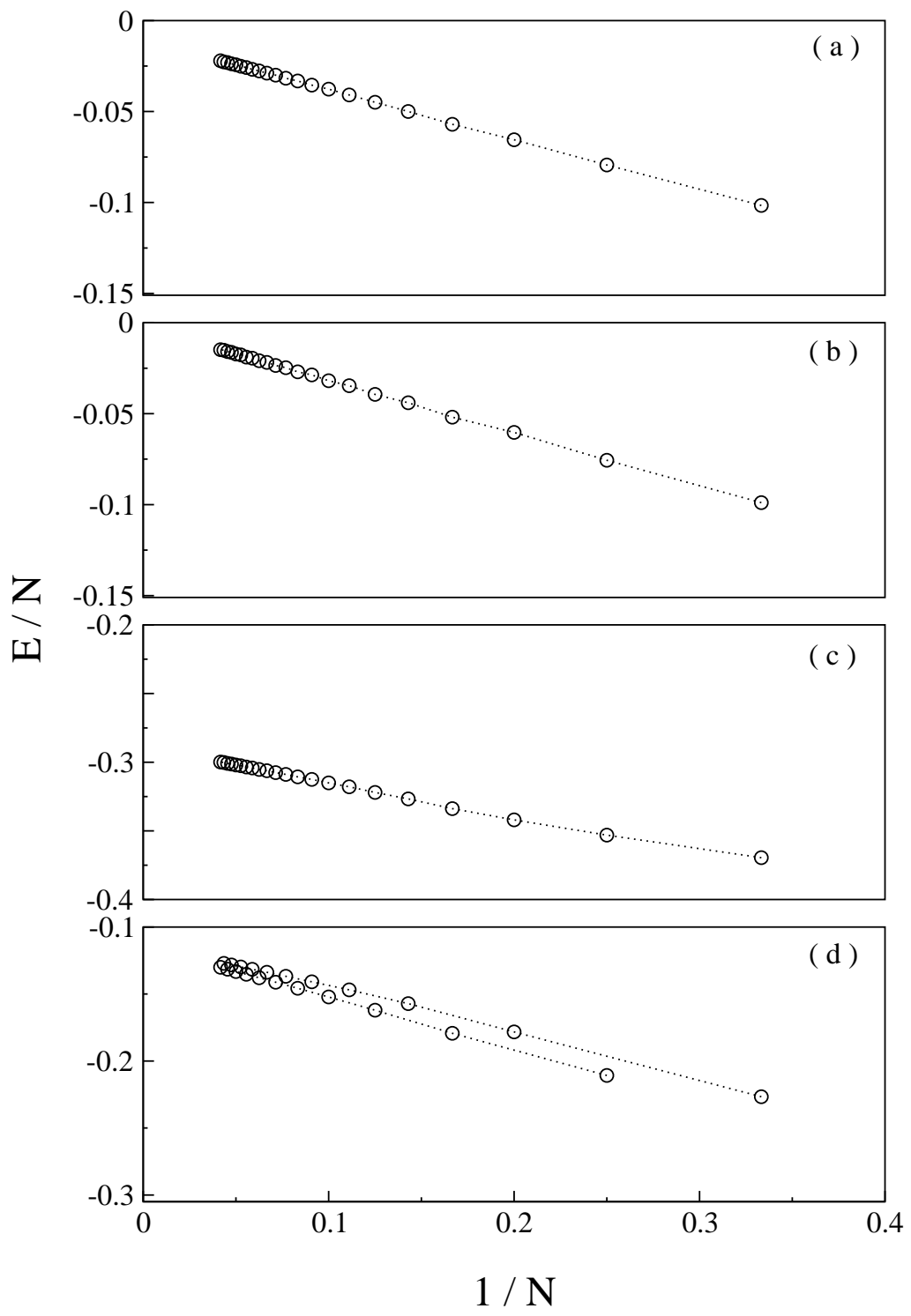

Figure 4: Convergence of energy per unit cell for polyacene oligomers, in the DMRG calculation, for (a) cis, $\delta=0.01$, (b) trans, $\delta=0.01$, (c) cis, $\delta=0.1$, (d) trans, $\delta=0.1$. 


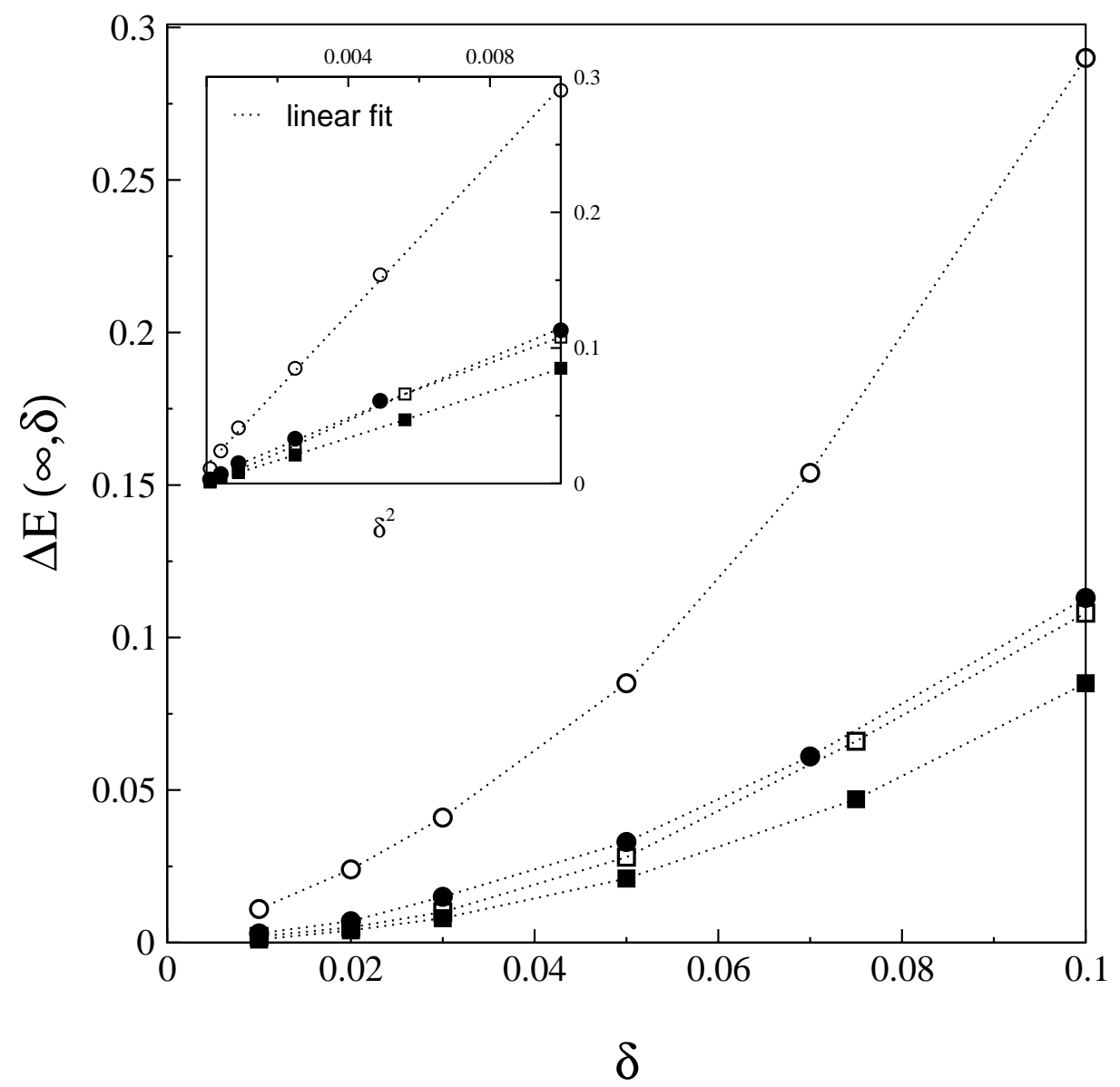

Figure 5: Stabilization energy for dimerization, as a function of $\delta$, the distortion. Circles and squares are for PPP and Hückel calculations and open and closed symbols stand for $c i s$ and trans forms of polyacene respectively. The inset figure shows the linear dependence of the stabilization energy when against $\delta^{2}$. 


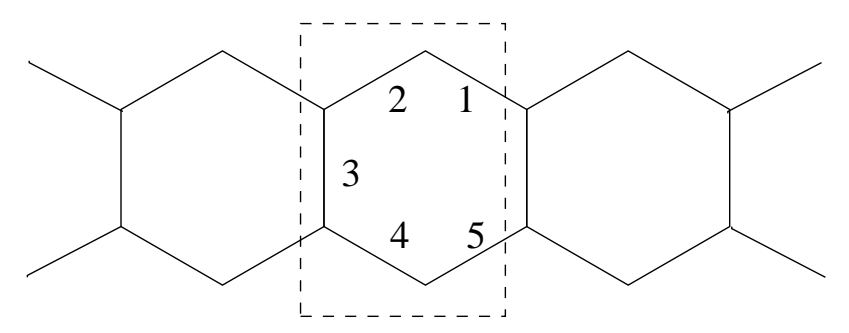

( a )

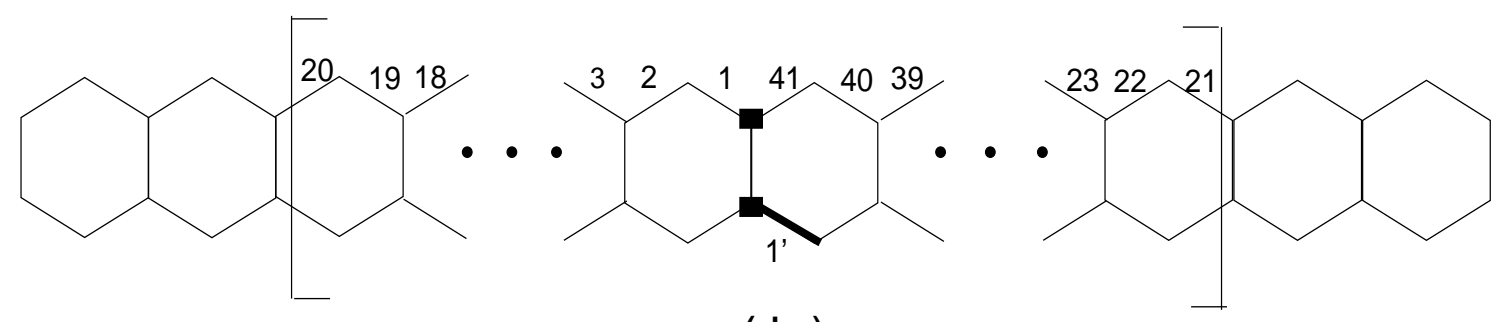

(b)

Figure 6: (a) Unitcell of polyacene showing the different bonds, (b) numbering of bonds in polyacene chain. The new right bond indicated by a thick line is the reference bond for bond-bond correlation. The new sites are indicated by filled squares. The square brackets indicate the part of the system over which periodic boundary conditions are applied to calculate bond structure factors. 


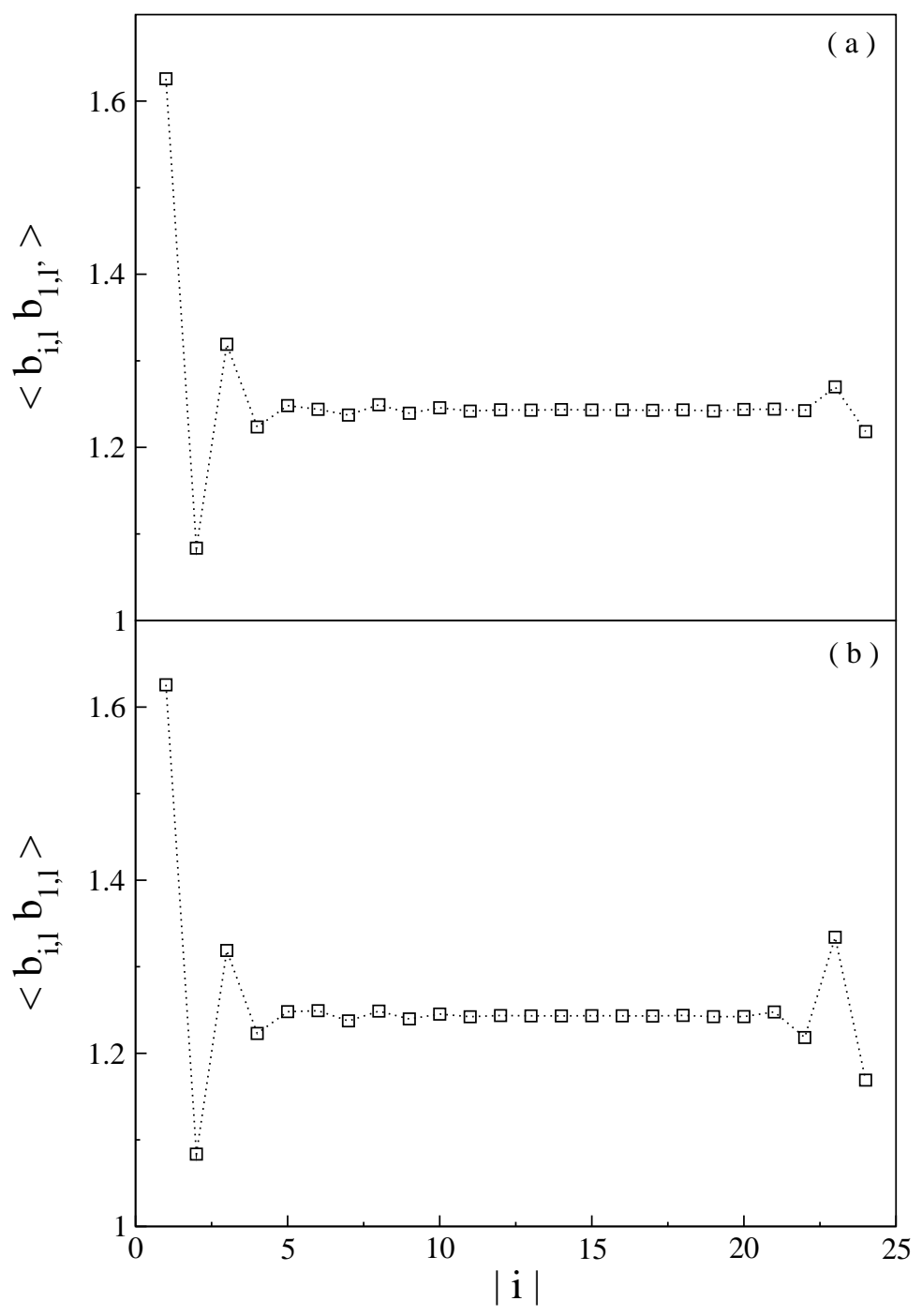

Figure 7: Bond-bond correlation as a function of the seperation between bonds, between the bond shown in bold in Fig. $6 \mathrm{~b}$ and the bonds in (a) the upper chain and (b) the lower chain of the left block. 


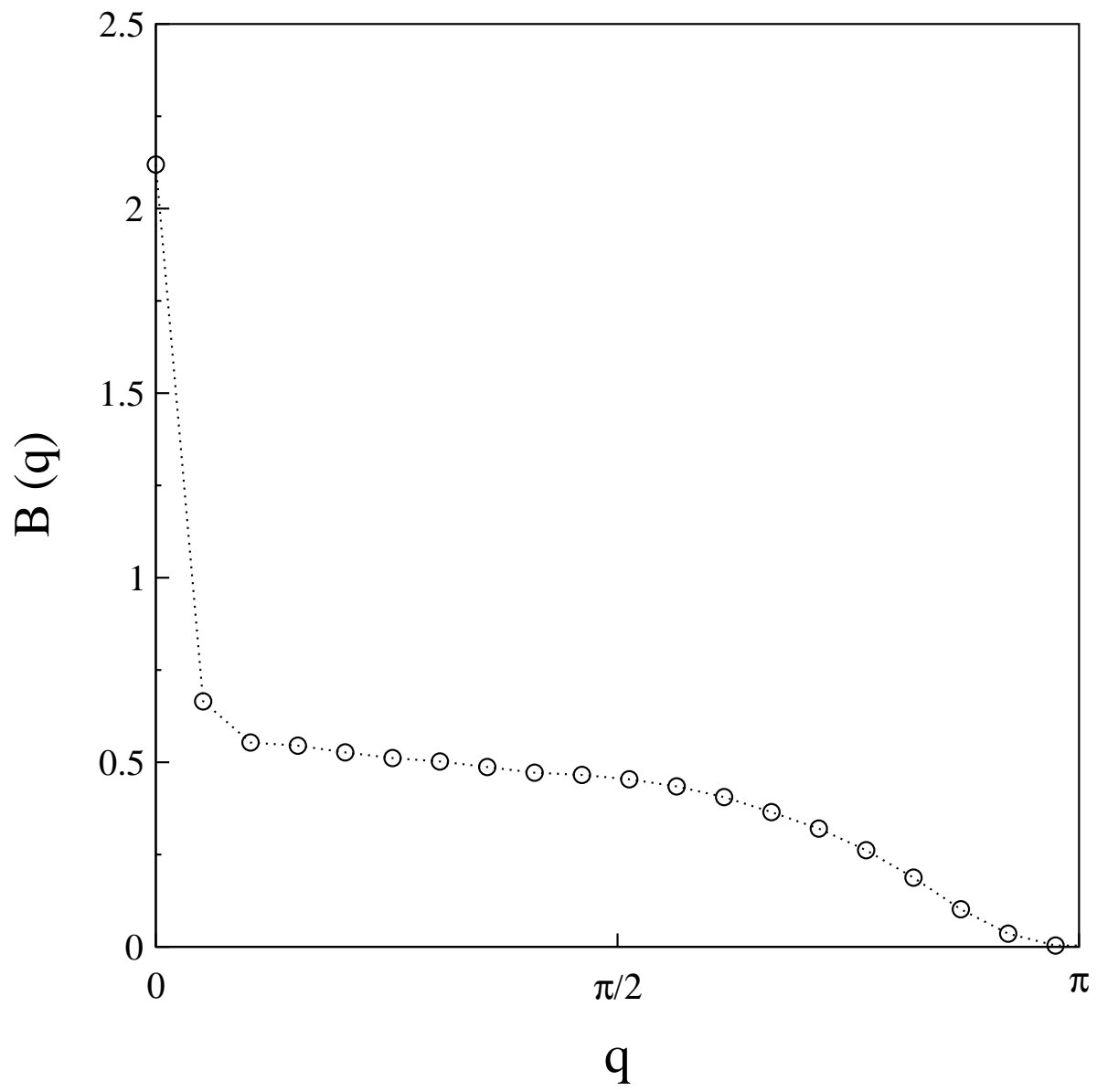

Figure 8: Bond structure factor corresponding to the bond-bond correlations shown in Fig. 7. 


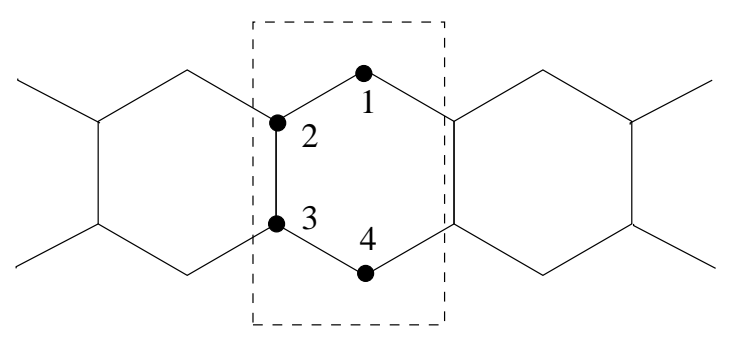

( a )

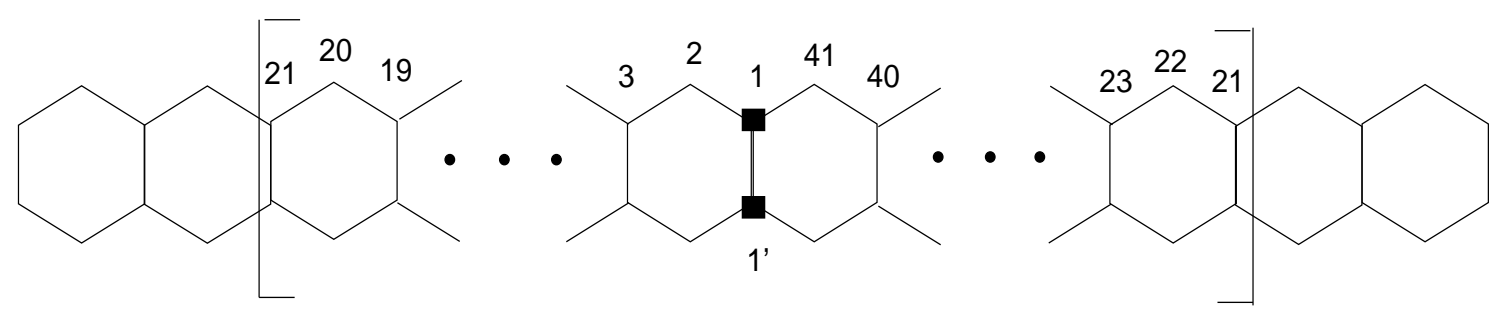

(b)

Figure 9: (a) Unitcell of polyacene showing the different sites, (b) numbering of sites in polyacene chain. The new sites are indicated by filled squares. The square brackets indicate the part of the system over which periodic boundary conditions are applied to calculate spin and charge structure factors. 


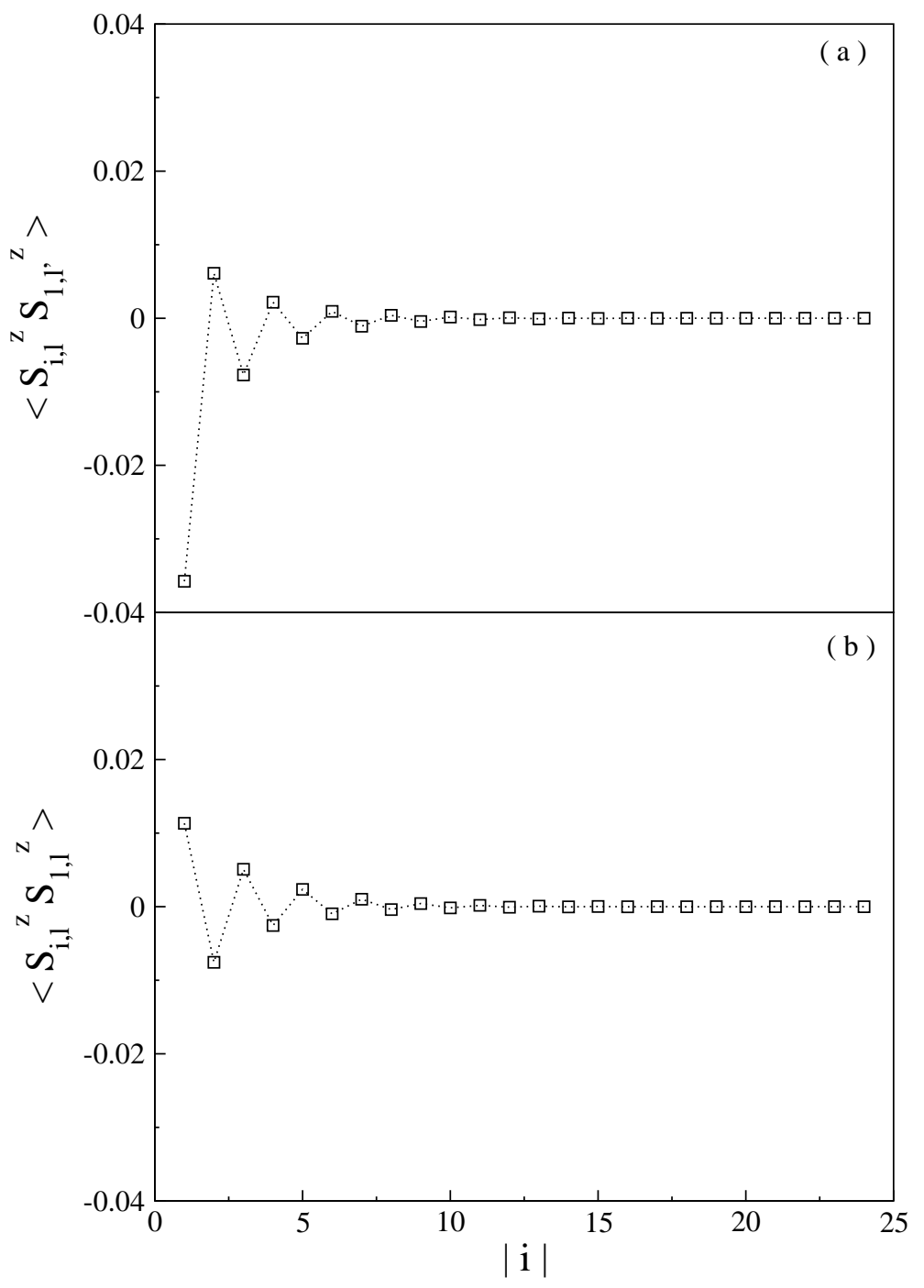

Figure 10: Spin-spin correlation as a function of the seperation between the new site in the right block and sites on (a) the upper chain and (b) the lower chain of the left block. 


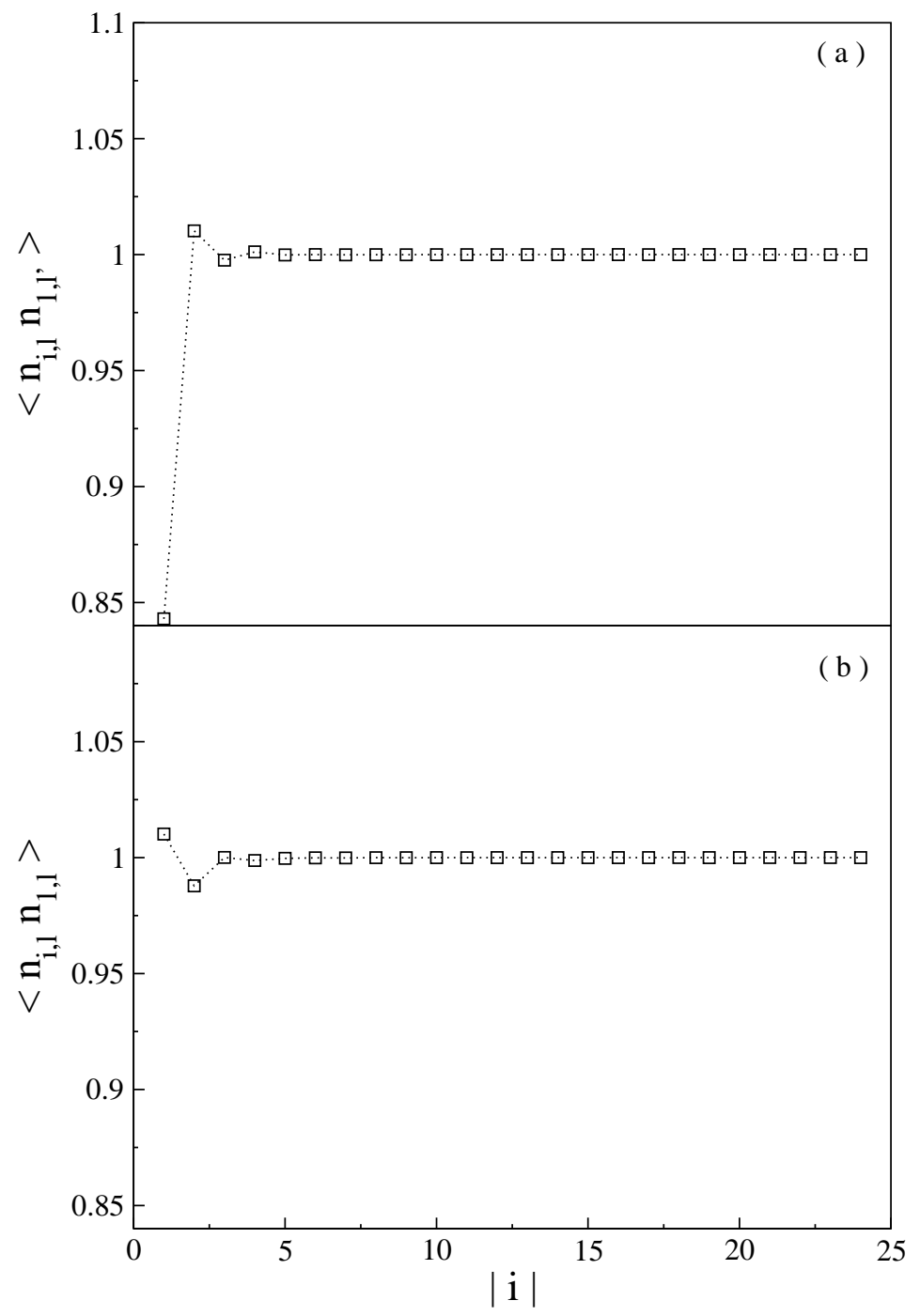

Figure 11: Charge-charge correlation as a function of the seperation between the new site in the right block and sites on (a) the upper chain and (b) the lower chain of the left block. 


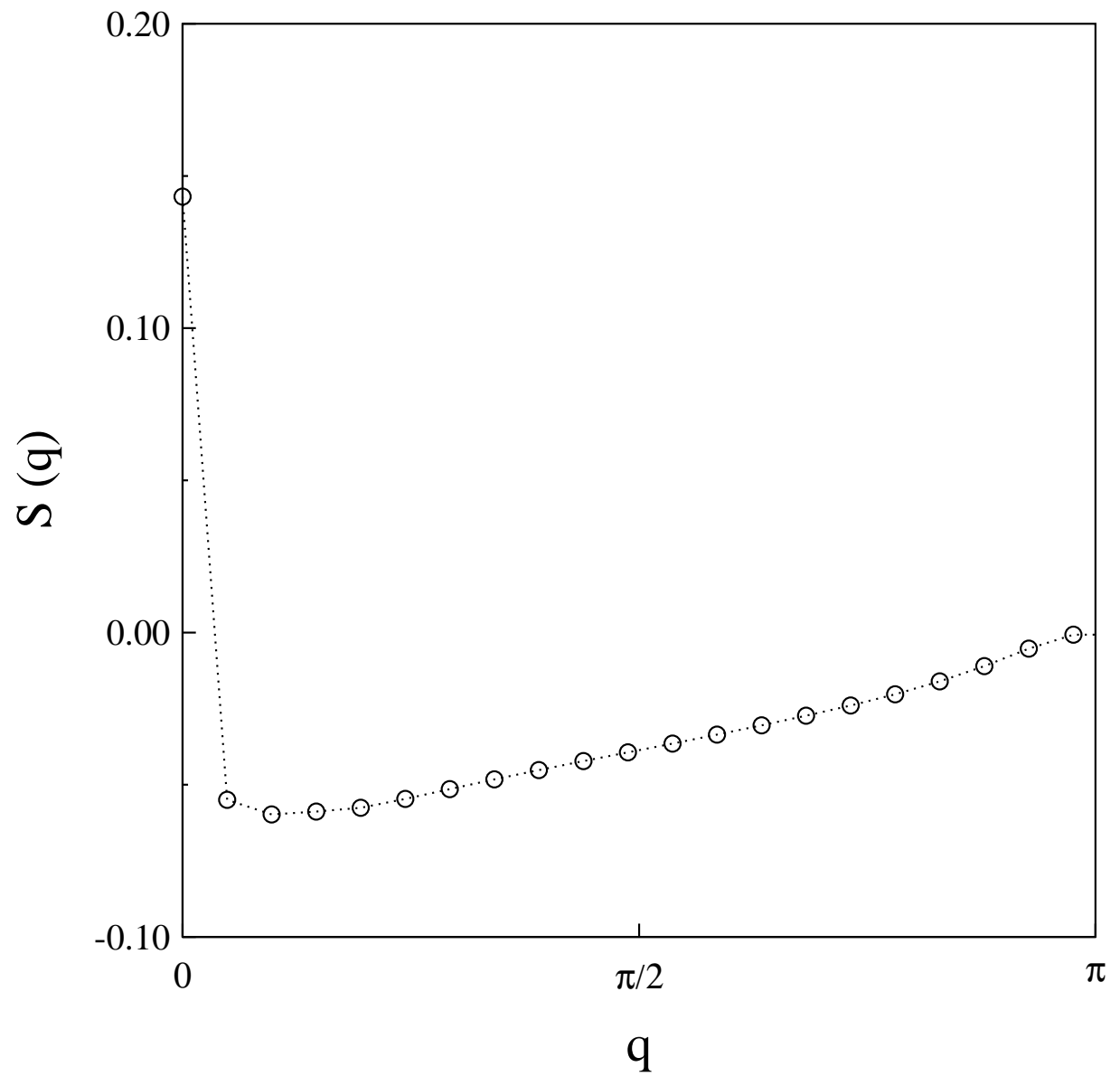

Figure 12: Spin structure factor obtained from the spin-spin correlation function shown in Fig. 10. 


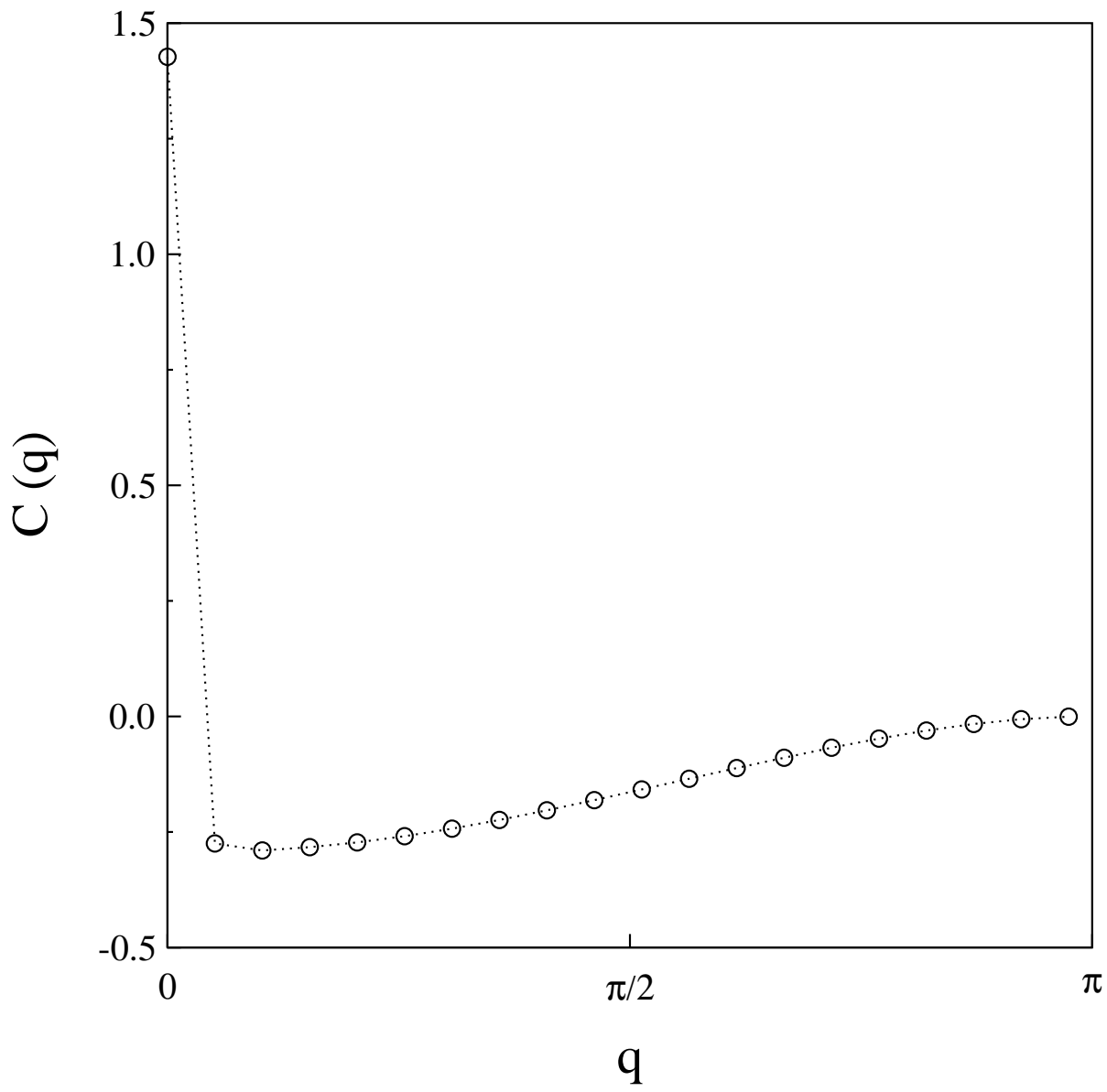

Figure 13: Charge structure factor obtained from the charge-charge correlation function shown in Fig. 11. 